\title{
Optimum apodization profile for chirped fiber Bragg gratings based chromatic dispersion compensator
}

\author{
Y. T. Aladadi', A. F. Abas ${ }^{1,2^{*}}$ and M. T. Alresheedi ${ }^{1}$
}

\begin{abstract}
In this paper, we optimized the apodization profile to improve the dispersion compensation performance of the chirped fiber Bragg gratings (CFBGs). Half tanh half uniform (HTHU), half exponential half uniform (HEHU), and half hamming half uniform (HHHU) apodization profiles were evaluated at $2000 \mathrm{~nm}$ wavelength. At this wavelength, Hollow-Core Photonic Crystal Fiber (HC-PCF) will be the targeted fiber for the future implementation. In this work, our aim is to obtain the dispersion compensator design with minimum average group delay ripple (GDR) and maximum Full Width Half Maximum (FWHM) bandwidth. The result shows that the best FWHM bandwidth is obtained by using HTHU profile that is approximately $96.27 \%$. In term of GDR, all apodization profiles show similar performance.
\end{abstract}

Keywords: Chromatic dispersion compensator, Chirped FBG, HC-PCF, Optimization, Apodization

\section{Background}

Exploring $2000 \mathrm{~nm}$ transmission window in optical communication system is one of important solutions to increase the bandwidth. To explore this transmission window, several measures need to be taken in optimizing dispersion compensation for this window. HollowCore Photonic Crystal Fiber (HC-PCF) was introduced as the transmission fiber, which is dominated by waveguide dispersion [1]. The Group Velocity Dispersion (GVD) of HC-PCF can be compensated at a given wavelength by scaling the fiber dimensions. However, this method is costly and complex. The dispersion slope of HC-PCFs is very steep. Consequently the range of zero dispersion wavelengths is very small. This means that without Chromatic Dispersion Compensator (CDC), HCPCF can only support a small number of Dense Wavelength Division Multiplexing (DWDM) channels. One of the methods to compensate HC-PCF's GVD is to use apodized Chirped Fiber Bragg Gratings (CFBG) [2]. Several researches focused on optimizing the apodization profile

\footnotetext{
* Correspondence: aabas@ksu.edu.sa

${ }^{1}$ Department of Electrical Engineering, College of Engineering, King Saud University, Riyadh 11421, Saudi Arabia

${ }^{2}$ Wireless and Photonics Research Center, Department of Computer and Communication Systems Engineering, Faculty of Engineering, Universiti Putra Malaysia, 43400 UPM, Serdang, Selangor, Malaysia
}

to improve the performance of $\mathrm{CDC}$ at $1.55 \mu \mathrm{m}$ wavelength [3-5]. Selection of the best parameters for the CFBG such as grating length, chirp period and index variation is very important. Apodization is a crucial procedure to reduce the group delay ripple that influences by chirped FBG dispersion characteristics [2, 6, 7]. The worst effect of apodization is losses in the bandwidth, which is very crucial.

In this paper, a simulation by using Matlab is conducted to evaluate three apodization profiles namely Half Tanh Half Uniform (HTHU), Half Exponential Half Uniform (HEHU), and Half Hamming Half Uniform (HHHU) profiles. The average group delay ripple and the bandwidth's variation are monitored to determine the optimum CDC performance at $2000 \mathrm{~nm}$ wavelength.

\section{Methods}

In order to design a CFBG with specified reflectivity and bandwidth, we use an objective reflectivity response as a reference for the designing process. We set $100 \%$ reflectivity, and $0.8 \mathrm{~nm}$ Full Width Half Maximum (FWHM) bandwidth as the target as shown in Eq. 1: 


$$
R_{O b j}=\left\{\begin{array}{rr}
1 & 2019.6 \mathrm{~nm} \leq \lambda \leq 2020.4 \mathrm{~nm} \\
0 & \text { elsewhere }
\end{array}\right.
$$

The optimum value of refractive index modulation amplitude, $\Delta n$, CFBG length, $L_{g}$, and the chirp parameter, $C$, is obtained by using the error estimation equation (Eq. 2) where error, $E$ is defined as

$$
E=\sum_{i=1}^{N}\left(R_{O b j}\left(\lambda_{i}\right)-R_{S i m}\left(\lambda_{i}\right)\right)^{2}=0, i=1,2, \ldots, N
$$

where $\lambda_{i}$ is the discrete set of wavelengths, and $R_{\text {sim }}$ is the simulated reflectivity.

Referring to Eq. (2), our simulation shows that the parameters that give the minimum $E$ are $L_{g}=108 \mathrm{~mm}$, $\Delta n=9 e^{-5}$ and $C=-0.23 e^{-1} \mathrm{~nm} / \mathrm{cm}$.

Figure 1 shows the simulated reflectivity response (blue) of a non-apodized CFBG, and the corresponding group delay (red). It is shown that the reflectivity nearly reaches $100 \%$ and the $3-\mathrm{dB}$ bandwidth is about $0.8 \mathrm{~nm}$. The slope of the group delay indicates the amount of chromatic dispersion that can be compensated by our CDC that is about $-1500 \mathrm{ps} / \mathrm{nm}$. The value of group delay ripple (GDR) is too large, which is around $\pm 30 \mathrm{ps}$. Therefore, there is an urgent need to reduce GDR while maintaining the bandwidth as optimum as possible.

\section{Results and discussion}

To suppress the GDR, several apodization profiles have been tested [8]. In this paper, we optimize the following apodization profiles in which we consider the bandwidth to be the effective element as GDR.

Half Tanh- half uniform profile (HTHU):

$$
\overline{\delta n}_{\text {eff }}(z)= \begin{cases}\frac{\tanh h^{2}\left(2 a \frac{z}{L_{g}}\right)}{\tanh ^{2}(a)}, & 0 \leq z<\frac{L_{g}}{2} \\ 1 \quad & , \frac{L_{g}}{2} \leq z \leq L_{g}\end{cases}
$$

Half Exponential-half uniform profile (HEHU):

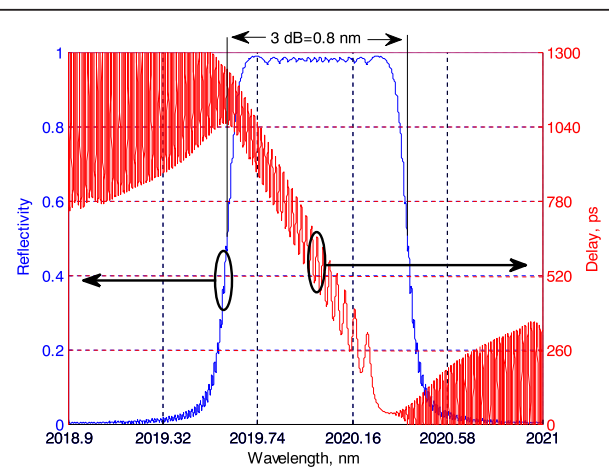

Fig. 1 The simulated reflectivity (blue), the group delay (red)

$$
\overline{\delta n}_{\text {eff }}(z)= \begin{cases}\exp \left(-a \frac{\left(z-\frac{L_{g}}{2}\right)^{4}}{L_{g}^{4}}\right) & , 0 \leq z<\frac{L_{g}}{2} \\ 1 \quad, \frac{L_{g}}{2} \leq z \leq L_{g}\end{cases}
$$

Half Hamming-half uniform profile (HHHU):

$$
\overline{\delta n}_{\text {eff }}(z)= \begin{cases}\frac{\left(1+a \cos \left(\pi \frac{2 z-L_{g}}{L_{g}}\right)\right)}{(1+a)}, & 0 \leq z<\frac{L_{g}}{2} \\ 1 & , \frac{L_{g}}{2} \leq z \leq L_{g}\end{cases}
$$

Sharpness parameter $a$ is used to control the sharpness of the apodization profile.

Reflectivity and group delay vary with the refractive index change, $\overline{\delta n}_{\text {eff }}(z)$. Eq. 3, Eq. 4 and Eq. 5, show that the sharpness parameter $a$ is the variable that determines the value of $\overline{\delta n}_{\text {eff }}(z)$. Therefore, it can also be used to optimize the reflectivity and GDR. Varying parameter $\alpha$ will vary both reflectivity FWHM bandwidth, and GDR. However, minimizing GDR will be accompanied by the loss of reflectivity bandwidth. Figure 2 (a) and (b) respectively show the effect of implementing HTHU profile (Eq. 3) with $\alpha=1$ to the reflectivity bandwidth and GDR. It can be noticed that the bandwidth reduces
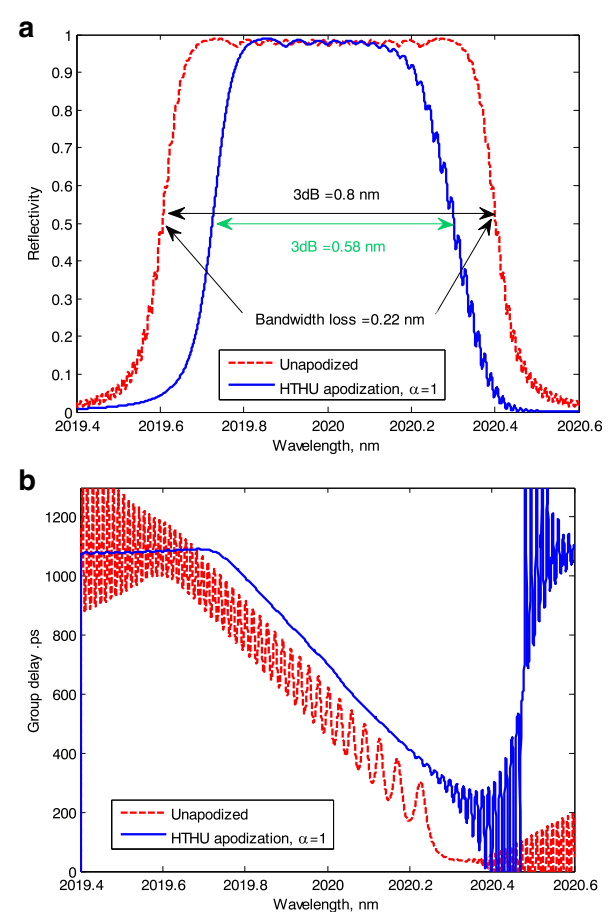

Fig. 2 Apodized and unapodized (a) reflectivity and (b) group delay 
significantly as the targeted GDR is achieved. This tradeoff shows that the optimization of the apodization profile is very important to obtain optimum performance.

Selecting the optimum point of $\alpha$ solves a tradeoff between the maximum FWHM bandwidth and minimum GDR. Thus, we rely on a new method to manage this conflict. This method depends on the relationship between the normalized apodized mean GDR and the apodized FWHM bandwidth. Equations (6) and (7) represent the normalized GDR, $F_{\text {ripple }}$ and normalized FWHM bandwidth, $F_{\text {bandwidth }}$, where $A_{\text {apod }}$ and $A_{\text {unapod }}$ are the apodized and unapodized peak to peak GDR amplitude respectively. $B W_{\text {apod }}$ and $B W_{\text {unapod }}$ are the apodized and unapodized FHWM bandwidth respectively.

$$
\begin{aligned}
& F_{\text {ripple }}=\frac{A_{\text {apod }}(a)}{A_{\text {unapod }}} \\
& F_{\text {bandwidth }}=\frac{B W_{\text {apod }}(a)}{B W_{\text {unapod }}}
\end{aligned}
$$

The relationship between $F_{\text {ripple }}$ and $F_{\text {bandwidth }}$ for the HEHU apodization profile (Eq. 4) are shown in Fig. 3.

To obtain the optimum value of $\alpha$, the distance $d$ which is defined by Eq. (8) is evaluated.

$$
d(a)=\sqrt{\left(\left(F_{\text {ripple }}(a)\right)^{2}+\left(1-\left(F_{\text {bandwidth }}(a)\right)^{2}\right.\right.}
$$

Distance $d$ is measured from the point $F_{\text {ripple }}=0$ and $F_{\text {bandwidth }}=1$, to any point on the curve, as depicted in Fig. 3.

The optimum point of $\alpha$ can be obtained at the minimum value of d, denoted as $d_{\text {min }}$. Figure 4 (a), Fig. 4 (b) and Fig. 4 (c) show the values of $d$ at different value of sharpness parameter for different apodization profile. $d_{1}$, $d_{2}$ and $d_{3}$ are the shortest distance for HTHU, HEHU and HHHU profiles respectively. These results occur at $a=13$ for HTHU profile, $a=47$ for HEHU and $a=1$ for HHHU $\left(d_{1}=0.1209, d_{2}=0.1935, d_{3}=0.2556\right)$.

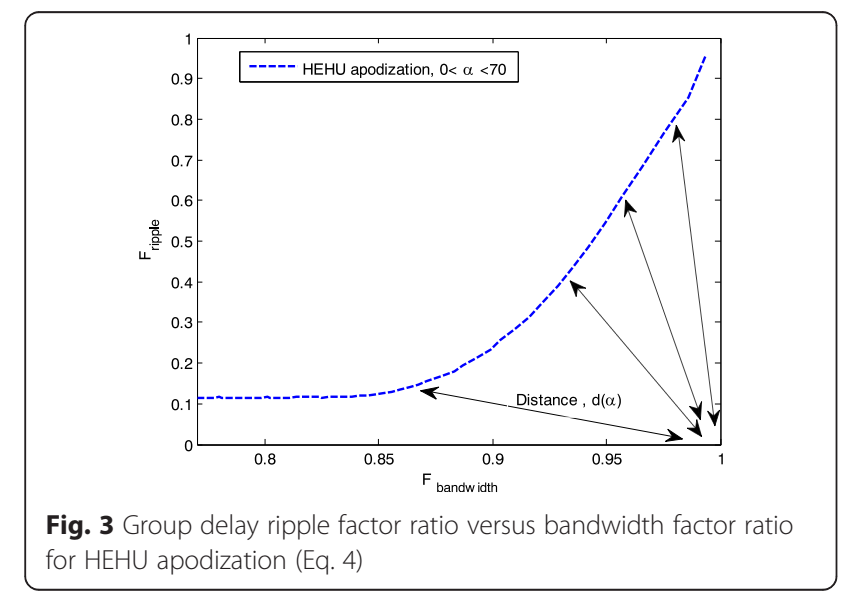

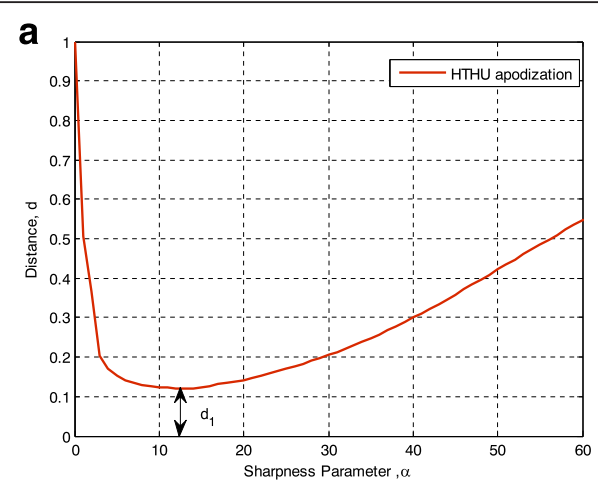
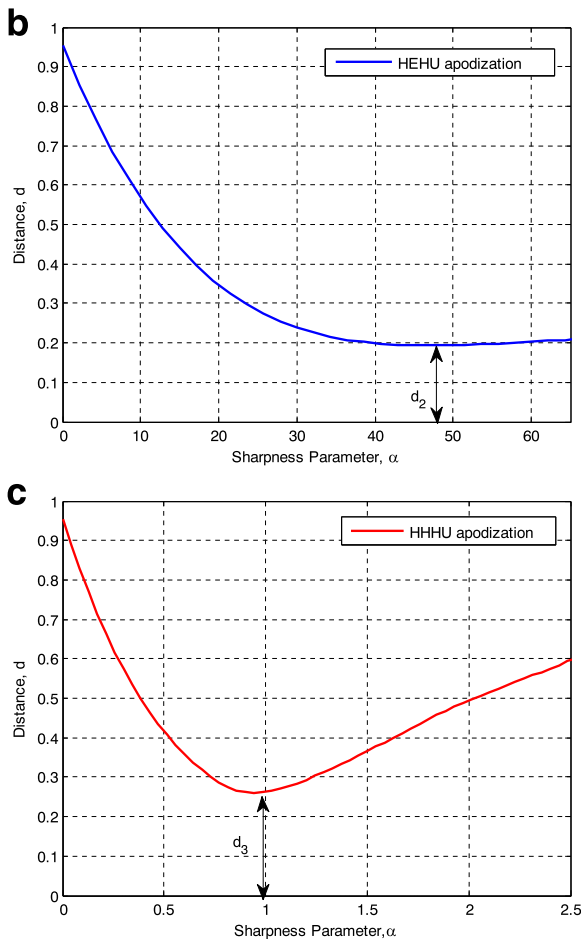

Fig. 4 The distance, $d$, versus the sharpness parameter, a for (a) HTHU profile, (b) HEHU profile, (c) HHHU profile

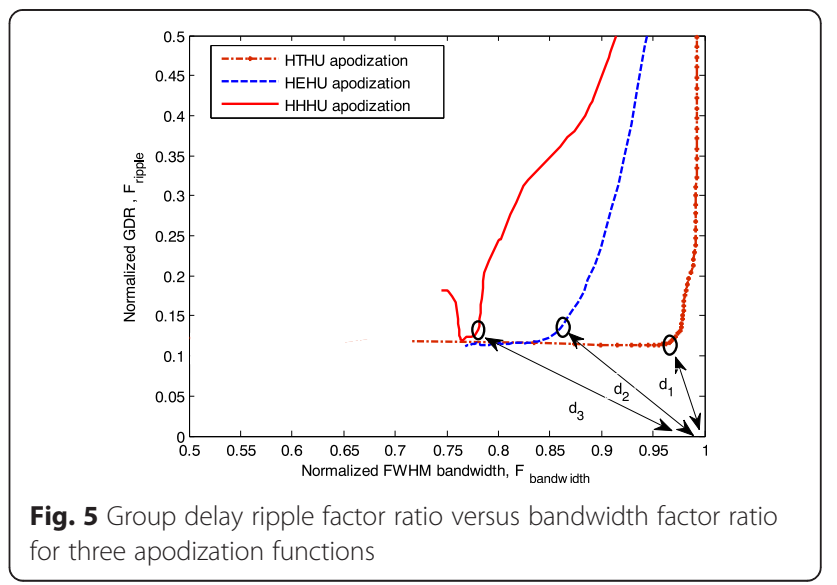




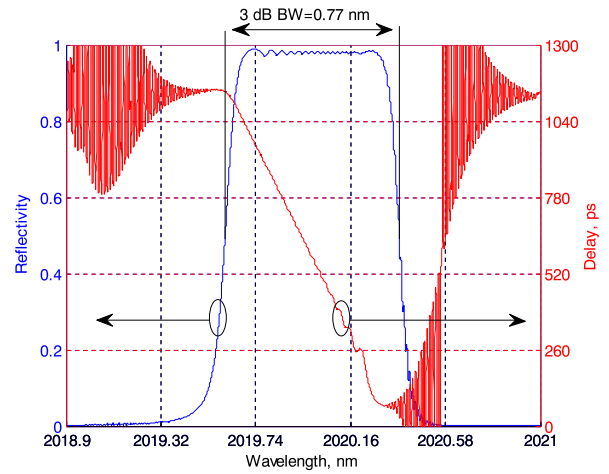

Fig. 6 The simulated reflectivity (blue color), the group delay (red color) with optimized HTHU apodization profile

Figure 5 shows the relationship between $F_{\text {bandwidth }}$ and $F_{\text {ripple }}$ for HTHU, HEHU and HHHU apodization profiles during the optimization process. All apodization profiles manage to reach the minimum value of $F_{\text {ripple }}$ of around 0.12 . In contrast, different values of bandwidth are achieved for different apodization profile. The FWHM bandwidth for HTHU profile is the closest to the targeted value $(0.8 \mathrm{~nm})$, which is approximately $96.27 \%$. HEHU profile obtained $85.56 \%$ and $77.71 \%$ is recorded for HHHU profile. Therefore, we decided that HTHU is the best among the 3 optimized apodization profiles, and is the best to be used in the design of the CFBG.

Figure 6 shows the reflectivity response of CFBG and corresponding group delay after applying the HTHU apodization profile with $\alpha=13$. In comparison to Fig. 1 (unapodized case), the FWHM bandwidth is reduced by around $0.03 \mathrm{~nm}$. This reduction is acceptable as it may not significantly affect the characteristic of the system. Figure 7 and Fig. 8 show the reflectivity responses and corresponding group delay after applying HEHU and HHHU apodization profiles respectively. The values of $\alpha$ are 47 and 1 for HEHU and HHHU apodization profiles respectively. The percentage bandwidth loss in case of HEHU and HHHU are $14.5 \%(0.115 \mathrm{~nm})$ and $22 \%$

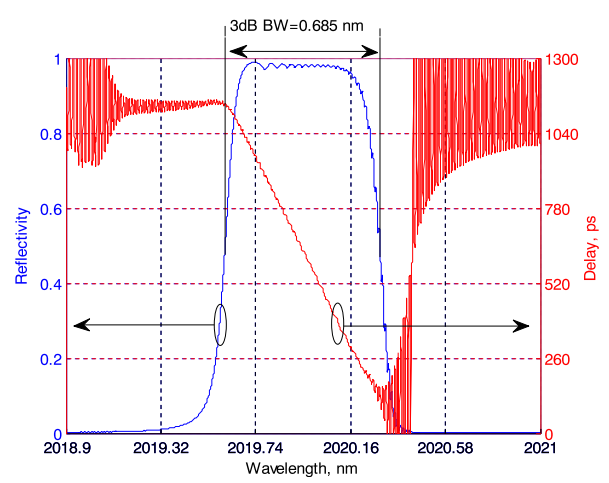

Fig. 7 The simulated reflectivity (blue color), the group delay (red color) with optimized HEHU apodization profile

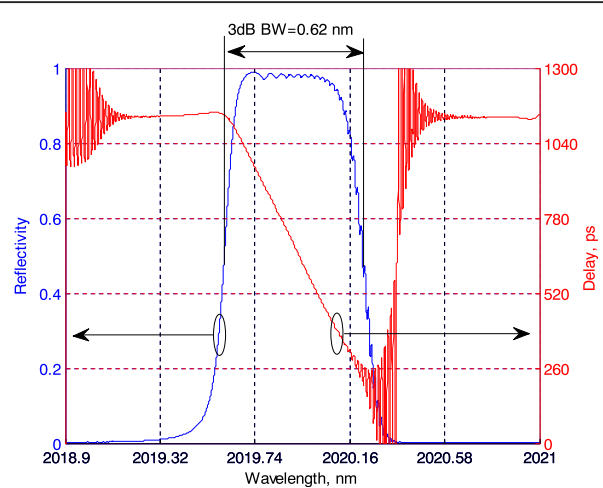

Fig. 8 The simulated reflectivity (blue color), the group delay (red color) with optimized $\mathrm{HHHU}$ apodization profile

$(0.18 \mathrm{~nm})$ respectively. On the other hand, with the best optimized apodization profiles, the GDR is reduced to the minimum extent. These results show that our target to design a CFBG that can become the CDC with optimum performance has been achieved.

\section{Conclusions}

HTHU profile is found to be the best apodization profile in designing high performance CDC. The optimization method used in this work is a good technique to evaluate and optimize the apodization profile. Our results show that by using HTHU profile, CDC working at $2000 \mathrm{~nm}$ wavelength range can be realized with optimum performance. Apart from the tested wavelength, with minimum modification, this optimization method can also be used for other bands. This report may become very important for the CDC researchers especially those who have interest in exploring the future $2000 \mathrm{~nm}$ bands.

\section{Competing interests}

The authors declare that they have no competing interests.

\section{Authors' contributions}

YTA formulated minimum distance optimization equation and carried out all the simulations. AFA proposed the concept of the study, involves in data analysis and overall manuscript preparation. MTA formulated the error estimation equation and all optimized apodization profiles. All authors read and approved the final manuscript.

\section{Acknowledgment}

The authors would like to extend their sincere appreciation to the Deanship of Scientific Research at King Saud University for supporting this work through RG-1437-008 Research Group Fund.

Received: 26 October 2015 Accepted: 12 May 2016

Published online: 27 June 2016

\section{References}

1. Russell, PSJ: Photonic-crystal fibers. J Lightwave Technol. 24, 4729-4749 (2006)

2. Ennser, K, Zervas, MN, Laming, R: Optimization of apodized linearly chirped fiber gratings for optical communications. Quantum Electron. 34, 770-778 (1998)

3. Mohammed, NA, Ali, TA, Aly, MH: Performance optimization of apodized FBG-based temperature sensors in single and quasi-distributed DWDM systems with new and different apodization profiles. AlP Advances. 3, 122-125 (2013) 
4. Zhang, H: A novel method of optimal apodization selection for chirped fiber Bragg gratings. Optik Int J Light Electron Optics. 125, 1646-1649 (2014)

5. Osuch, T, Markowski, K, Jedrzejewski, K: Numerical model of tapered fiber Bragg gratings for comprehensive analysis and optimization of their sensing and strain-induced tunable dispersion properties. Appl Optics. 54, 5525-5533 (2015)

6. Fernandez, P, Aguado, J, Blas, J, Duran, R, de Miguel, I, Duran, J, Lorenzo, R, Abril, E: Analysis and optimisation of the apodisation sharpness for linearly chirped dispersion compensating gratings. IEE Proc Optoelectron. 151, 69-73 (2004)

7. Williams, J, Bennion, I, Doran, N: The design of in-fiber Bragg grating systems for cubic and quadratic dispersion compensation. Optics Commun. 116, 62-66 (1995)

8. Erdogan, T: Fiber grating spectra. Lightwave Technol. 15, 1277-1294 (1997)

\section{Submit your manuscript to a SpringerOpen ${ }^{\circ}$ journal and benefit from:}

- Convenient online submission

- Rigorous peer review

- Immediate publication on acceptance

- Open access: articles freely available online

- High visibility within the field

- Retaining the copyright to your article

Submit your next manuscript at $\boldsymbol{s p r i n g e r o p e n . c o m ~}$ 